\title{
A STUDY OF DEEP VEIN THROMBOSIS IN SURGICAL PRACTICE
}

\author{
Pritam Pritish Patnaik1, Bhupati Bhusan Das², Niranjan Sahoo³, Sushanta Kumar Das, Charan Panda 5 , Mahammad Asfak Ahmed 6 \\ ${ }^{1}$ Resident, Department of General Surgery, MKCG Medical College \& Hospital, Ganjam, Berhampur. \\ ${ }^{2}$ Assistant Professor, Department of General Surgery, MKCG Medical College \& Hospital, Ganjam, Berhampur. \\ 3 Assistant Professor, Department of General Surgery, MKCG Medical College \& Hospital, Ganjam, Berhampur. \\ 4 Professor, Department of General Surgery, MKCG Medical College \& Hospital, Ganjam, Berhampur. \\ 5 Professor, Department of General Surgery, MKCG Medical College \& Hospital, Ganjam, Berhampur. \\ ${ }^{6}$ Senior Resident, Department of General Surgery, MKCG Medical College \& Hospital, Ganjam, Berhampur.
}

\section{ABSTRACT}

This was a prospective clinical study performed in the surgical department of MKCG Medical College and Hospital, Berhampur, Odisha, during the period from September 2013 to August 2015. Six hundred and forty eight patients who underwent surgical procedures $>1$ hour were included in this study to find the incidence of deep vein thrombosis (DVT) development postoperatively.

\section{AIMS AND OBJECTIVES}

Identifying the possible risk factors and to identify the subgroup of patients needing prophylaxis routinely after a surgical procedure.

\section{METHOD OF COLLECTION OF DATA}

Patients aged >15 years who underwent surgeries (APR, colostomies, abdominal surgeries, hernia repairs, MRM, thyroidectomies, perforation closure etc.) lasting for more than one hour under spinal, epidural and general anaesthesia. Patients unwilling to take part in the study, patients on anticoagulant therapy and patients previously diagnosed with DVT or VTE were excluded from the study.

\section{PROCEDURE}

Clinical examination was carried out in the postoperative period to look for limb pain and swelling. D-dimer levels and Doppler USG were used for diagnosis.

\section{RESULTS AND CONCLUSION}

The incidence of DVT was 1.23\% and the mean age group of the patients affected was 50.5 years. Male-to-female ratio was 1.28:1. OCP intake, smoking, higher BMI, major surgeries under GA and greater and postoperative immobilisation were important risk factors. Colonic surgeries had a greater chance of post-op DVT development. Duplex USG had a high sensitivity and specificity and thus can be considered the gold standard test for diagnosis of DVT, whereas D-dimer has low specificity and specificity thus not reliable for DVT diagnosis post-operatively.

\section{KEYWORDS}

DVT, VTE, D-dimer, Duplex USG.

HOW TO CITE THIS ARTICLE: Patnaik PP, Das BB, Sahoo N, et al. A study of deep vein thrombosis in surgical practice. J. Evolution Med. Dent. Sci. 2016;5(43):2673-2678, DOI: 10.14260/jemds/2016/626

\section{INTRODUCTION}

Venous thrombosis is defined as the formation of a semi-solid coagulum within the venous system. When it occurs in the superficial veins, it is known as thrombophlebitis and it is termed as phlebothrombosis when it occurs within the deep venous system.

Patients who undergo surgical procedures are at higher risk of developing Deep Vein Thrombosis (DVT).

A prospective study done by S.S.R Murthy. ${ }^{1}$ et al showed overall incidence of deep vein thrombosis in Indians was $1.3 \%$. P. O. Stein et al, concluded that the incidence of DVT in men aged 20 to 49 years was higher than in women of same age, while it was comparable among men and women $\geq 50$ years. $^{2}$

Financial or Other, Competing Interest: None.

Submission 13-04-2016, Peer Review 07-05-2016,

Acceptance 13-05-2016, Published 28-05-2016.

Corresponding Author:

Dr. Pritam Pritish Patnaik,

Room No.-70, Top Floor,

PG Hostel No. 02, MKCG Medical Campus,

Ganjam, Berhampur-760004,

Odisha.

E-mail: pritam1900@gmail.com

DOI: $10.14260 /$ jemds/2016/626

\section{AIMS AND OBJECTIVES}

1. To study the incidence of DVT in patients admitted to General Surgery ward with various surgical illnesses undergoing surgical management without any prophylactic anticoagulant therapy using Doppler studies.

2. To correlate the higher risk amongst the subgroup of patients with the duration of surgical procedures and other associated risk factors.

3. To identify the subgroup of patients and surgeries, in which routine DVT prophylaxis is to be recommended.

\section{Source of Data}

The study was conducted on 648 patients who underwent various operative procedures in the Dept. of General Surgery of MKCG Medical College and Hospital, Berhampur, during the period from September 2013 to August 2015.

\section{METHOD OF COLLECTION OF DATA}

Selection of Subject and Inclusion Criteria

All consecutive patients from both sex and with the following characteristics were included in the subject group,

1. Age $>15$ years. 
2. Surgery.

a. Under spinal, epidural or general anaesthesia.

b. Duration of surgery $>1$ hour.

Surgeries Included can be Broadly divided into Five Categories

I. Major abdominal surgeries for malignancies, like abdominoperineal resections, hemicolectomies, gastrectomies.

II. Abdominal surgeries for perforation of hollow viscera (Ileal, colonic and duodenal ulcer perforations)

III. Surgeries for intra-abdominal inflammatory pathology associated with intra-abdominal adhesions, cholecystitis and appendicitis.

IV. Other abdominal surgeries like vagotomy with GJ, inguinal hernias, ventral (Paraumbilical, incisional) hernias, Mesh rectopexies, etc.

V. Other surgeries like Thyroidectomies, Modified Radical Mastectomies, amputations, Surgeries for varicose veins, etc.

\section{Exclusion Criteria}

1. Patients unwilling to take part in the study.

2. Patients on anticoagulant therapy.

Patients previously diagnosed with DVT or venous thromboembolism (VTE).

A thorough systemic and local examination of lower limbs was done on all the patients.

Apart from routine blood investigations, special investigations like D dimer levels and Duplex Ultrasonography were done for all the patients.

\section{OBSERVATIONS}

\section{Incidence}

The incidence of DVT in this study was found to be = $8 / 648 \times 100=1.23 \%$.

\section{Age Distribution}

The minimum age of the studied age group was 20 years and maximum age was 78 years.

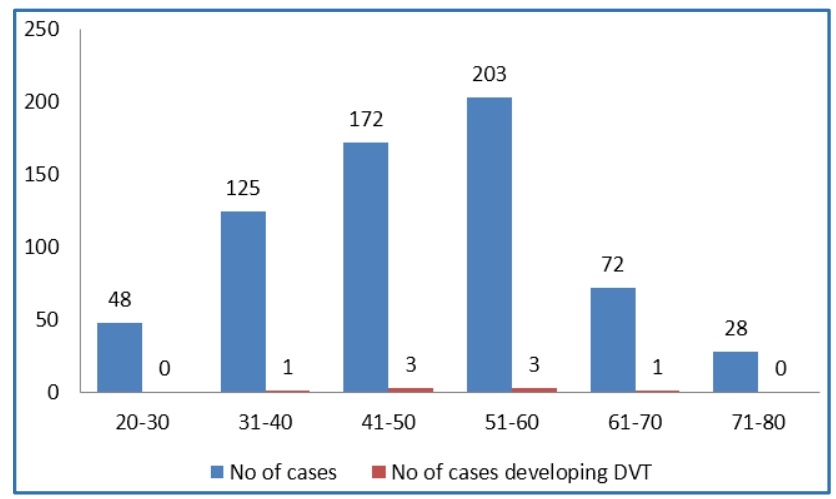

Fig. 1: Age Distribution of Study Population and those who Developed DVT

Mean age group of the study population was 47.945 years and the mean age group of the patients that developed DVT was 50.5 years.

\section{Sex Distribution}

The studied population comprised of $70 \%$ males and $30 \%$ of females. Out of the 8 patients who developed DVT, 6 (75\%) were males and $2(25 \%)$ were females.

The male-to-female ratio in development of postoperative DVT was 6/454:2/194=1.28:1.

\section{OCP Intake and DVT Correlation}

In the female study group, $15.46 \%$ were on OCPs and incidence of post-operative DVT among them was $3.33 \%$ as compared to $0.61 \%$ among the females, who were not on OCPs and overall female incidence of $1.03 \%$.

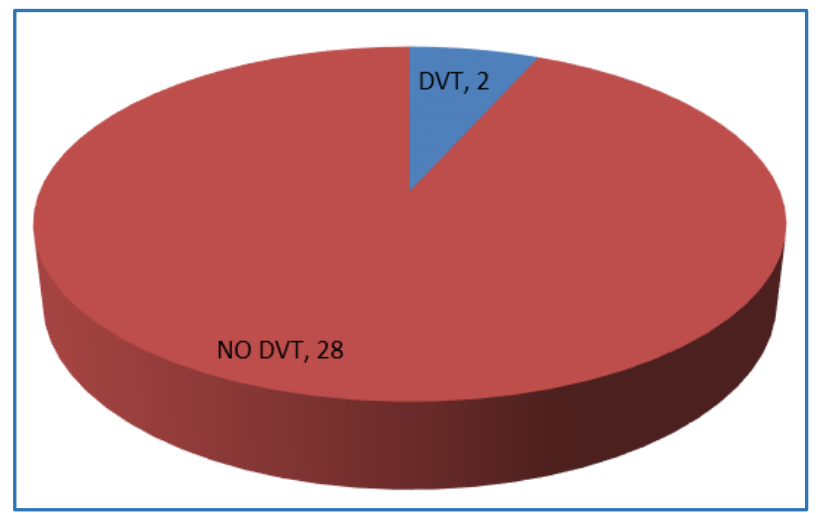

Fig. 2: Proportion of DVT in Females on OCP Intakes

Thus, females who consumed OCPs were at a greater risk of developing DVT post-operatively than those who did not use OCPs.

\section{Smoking and DVT Correlation}

Among the male patients, $54.63 \%$ were smokers and the incidence of DVT was $2.016 \%$ as compared to $0.48 \%$ among non-smokers. This incidence was also higher than the overall $1.32 \%$ incidence of DVT in males irrespective of the smoking status concluding smoking to be a significant risk factor.

\section{BMI and DVT}

The average BMI of the study population was 21.02 as compared to 29.875 of those who were diagnosed with DVT in the post-operative period.

Incidence of DVT in patients with BMI
Incidence of DVT in patients with BMI
$>25=6 / 55 \times 100=10.91 \%$.

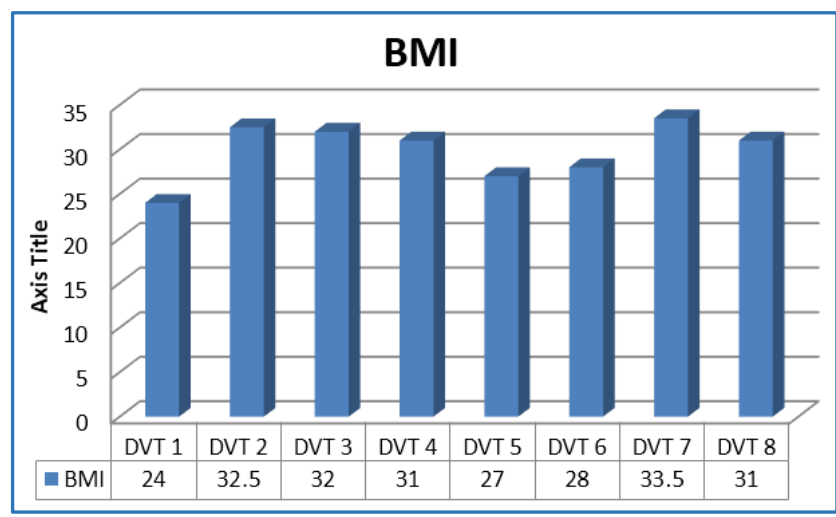

Fig. 3: BMI Distribution of Patients with DVT

Thus, it is evident that higher the BMI, greater is the risk of DVT development. 


\section{Malignancy and DVT}

Out of the 648 patients studied, 66 were cancer patients and the incidence of DVT was $4.54 \%$ in them as compared to $0.86 \%$ incidence in those who were negative for any malignancy. This incidence was also considerably higher than the overall incidence of $1.23 \%$ in the entire study population, which highlights the role of malignancy in favouring DVT development postoperatively.

\section{Hyperlipidaemia and DVT Development}

$13.9 \%$ of patients were hyperlipidaemic on routine blood investigations and the incidence of DVT was $6.67 \%$ in them in comparison with $0.36 \%$ DVT incidence in patients having a normal lipid profile, so hyperlipidaemia increases the risk.

\section{Duration of Surgery and DVT}

The average operating time in the entire study group was 1.99 hours as compared to a significantly higher value of 2.91 hours in patients who later on went on to develop DVT.

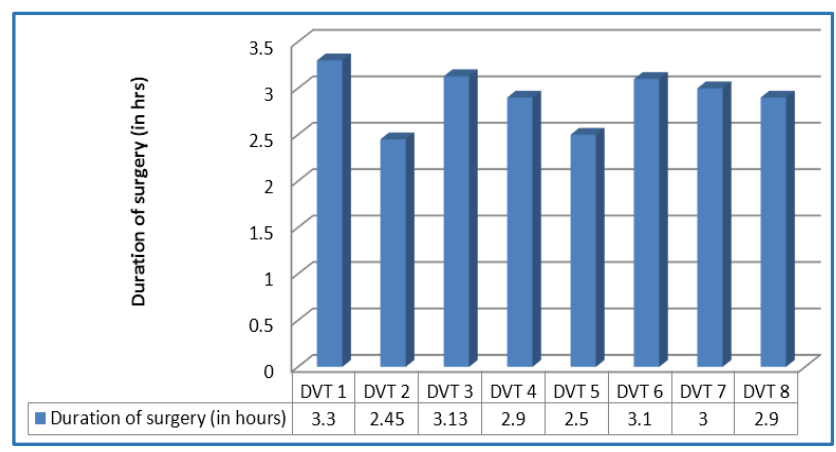

Fig. 4: Relation between Duration of Surgery and DVT Development

Relation of Type of Anaesthesia with DVT Development Out of 648 patients studied, 200 were given General Anaesthesia (GA), 408 spinal and rest 40 got epidural anaesthesia.

All the eight patients who developed DVT post-surgery, all were operated under general anaesthesia (Incidence of 4\%). The patients who received spinal and epidural anaesthesia did not show DVT post-operatively. Thus, GA increases the risk of DVT unlike spinal and epidural anaesthesia.

\section{Postoperative Immobilisation and DVT}

The average duration of immobilisation post-surgery was around 1.85 days, whereas in patients diagnosed with DVT it was around 2.875 days.

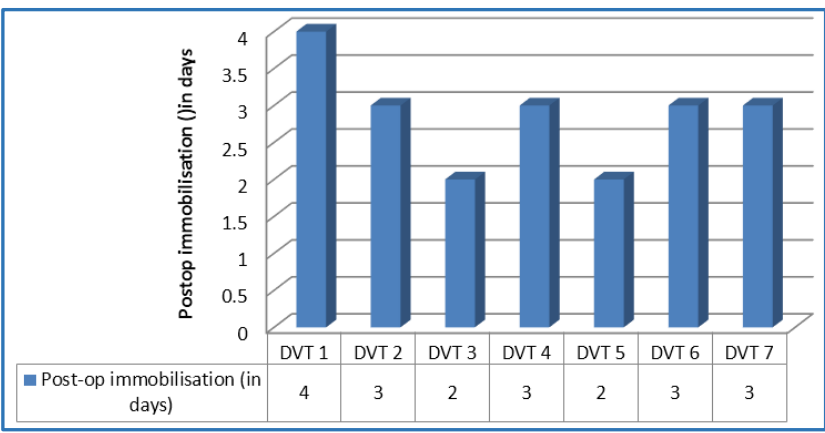

Fig. 5: Association of Postoperative Immobilisation and DVT

So, early ambulation after surgery can help minimise the risk of developing DVT.

\section{Types of Surgery Performed}

Out of 648 surgical procedures performed, (Varicose veins 6\%, Appendix - 10\%, Open Cholecystectomies - 8\%. Laparotomies for hollow viscus perforation and SAIO - $24.5 \%$. Gastrectomies, Palliative gastrojejunostomy or feeding jejunostomy - $6.5 \%$, Colonic surgeries - $6 \%$, inguinal hernia repair - $19.5 \%$. Ventral hernia repair - $9.5 \%$, thyroidectomy $6 \%$, MRM - 4\%).

Out of the eight patients who developed DVT, two underwent hemicolectomies, one left hemicolectomy for diverticular disease of descending colon and another right hemicolectomy for Crohn's disease presenting with multiple strictures, one female patient underwent MRM for carcinoma breast, one patient underwent resection and anastomosis for ileosigmoid compound volvulus, another underwent segmental ileocolic resection for ileocaecal tuberculosis, one had a transverse loop colostomy for advanced carcinoma rectum with obstruction, one had surgery for varicose veins (SFJ flush ligation with subfascial ligation of perforators) and the last patient underwent Abdominoperineal Resection (APR) for carcinoma rectum.

\section{Limb Pain and DVT}

Limb pain was a consistent symptom in all the patients who developed DVT postoperatively and had thus a sensitivity of $100 \%$, but a low specificity of $64.21 \%$.

\begin{tabular}{|c|c|c|}
\hline & DVT (+) & DVT (-) \\
\hline Limb pain (+) & 8 (True positive) & 229 (False positive) \\
\hline Limb pain (-) & 0 (False negative) & 411 (True negative) \\
\hline Total & $\mathbf{8}$ & $\mathbf{6 4 0}$ \\
\hline
\end{tabular}

\section{Limb Swelling and DVT}

\begin{tabular}{|c|c|c|}
\hline & DVT (+) & DVT (-) \\
\hline Limb swelling (+) & $\begin{array}{c}4 \text { (True } \\
\text { positive) }\end{array}$ & $\begin{array}{c}248 \text { (False } \\
\text { positive) }\end{array}$ \\
\hline Limb swelling (-) & $\begin{array}{c}4 \text { (False } \\
\text { negative) }\end{array}$ & $\begin{array}{c}392 \text { (True } \\
\text { negative) }\end{array}$ \\
\hline Total & $\mathbf{8}$ & $\mathbf{6 4 0}$ \\
\hline
\end{tabular}

Limb swelling was seen only in 4 out of the 8 patients who actually developed DVT, whereas it was falsely positive in 248 patients thereby having a low sensitivity and specificity of $50 \%$ and $61.25 \%$.

\section{Homans' Sign in DVT Cases}

Homans' sign, a clinical sign frequently used to suspect DVT in symptomatic patients yielded a low sensitivity of $50 \%$ and specificity of $51.875 \%$.

\begin{tabular}{|c|c|c|}
\hline & DVT (+) & DVT (-) \\
\hline Homans' sign (+) & $\begin{array}{c}4 \text { (True } \\
\text { positive) }\end{array}$ & $\begin{array}{c}308 \text { (False } \\
\text { positive) }\end{array}$ \\
\hline Homans' sign (-) & $\begin{array}{c}4 \text { (False } \\
\text { negative) }\end{array}$ & $\begin{array}{c}332 \text { (True } \\
\text { negative) }\end{array}$ \\
\hline Total & $\mathbf{8}$ & $\mathbf{6 4 0}$ \\
\hline
\end{tabular}

\begin{tabular}{|c|c|c|}
\hline D-Dimer & DVT (+) & DVT (-) \\
\hline D-Dimer (+) & 6 (True positive) & 86 (False positive) \\
\hline D-Dimer (-) & 2 (False negative) & 359 (True negative) \\
\hline Total & $\mathbf{8}$ & $\mathbf{6 4 0}$ \\
\hline \multicolumn{3}{|c|}{ D-Dimer in DVT } \\
\hline
\end{tabular}


The negative predictive value was high $99.44 \%$. The low specificity means that it cannot reliably exclude the individuals who actually do not have a DVT.

Duplex USG had a high sensitivity of $100 \%$ and high specificity of $98.90 \%$ in the diagnosis of DVT in the patients studied.

\begin{tabular}{|c|c|c|}
\hline Duplex USG & DVT (+) & DVT (-) \\
\hline Duplex USG(+) & 8 (True positive) & 5 (False positive) \\
\hline Duplex USG(-) & 0 (False negative) & 635 (True negative) \\
\hline Total & $\mathbf{8}$ & $\mathbf{6 4 0}$ \\
\hline \multicolumn{3}{|c|}{ Usefulness of Duplex USG in DVT } \\
\hline
\end{tabular}

So it can be considered as the gold standard test for the diagnosis of DVT.

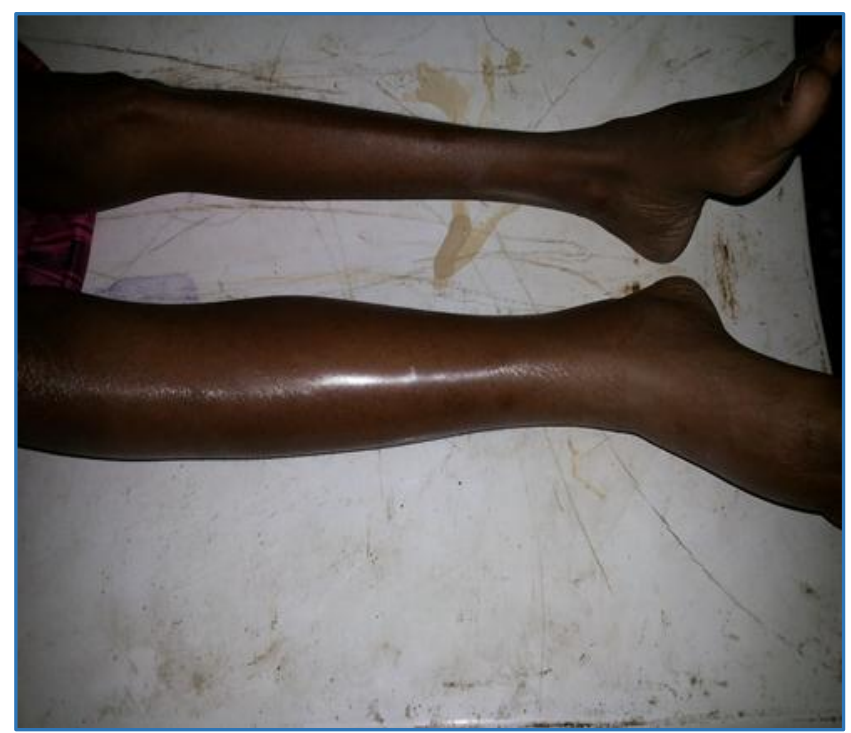

Figure showing Right LL DVT

\section{DISCUSSION}

\section{Incidence}

The incidence of post-op DVT in this study was $1.23 \%$, similar to an incidence of $1.6 \%$ as stated by Smith et al. ${ }^{3}$ The incidence of DVT is less among Asians as compared to Americans and Europeans possibly due to either genetic or environmental factors resulting in differences in the spectrum of mutations and congenital anticoagulant deficiencies according to Aiach et al. 4

\section{Age Distribution}

The mean age group studied was 47.9 years, whereas the average age of the patients who developed DVT postoperatively was higher being 50.5 years. This is in accordance with the study done by Rakesh et al. ${ }^{5}$ Old age is an acquired prothrombotic state and is associated with anatomic changes in the soleal veins causing pronounced stasis in the venous valve pockets.

\section{Sex Ratio}

The male-to-female ratio was $1.28: 1$. Study by Silverstein et $\mathrm{al}, 6$ also showed male preponderance (M:F=1.2:1).

However, there is lack of consistency in data on gender and DVT incidence and small differences are observed in either direction according to Legato. ${ }^{7}$

\section{OCPs and DVT}

The incidence of DVT in females on OCPs was four times than those who did not consume OCPs. So it carries a greater risk for DVT in the present study, which is similar to that reported by Vlieg $\mathrm{A}$ et al.8 Oestrogen in pharmacologic doses is associated with increase in blood viscosity, fibrinogen, plasma levels of factors VII and X and platelet adhesion and aggregation, which contribute to this thrombotic tendency.

\section{Smoking and DVT}

This study showed a higher incidence of DVT in smokers $(2.016 \%)$ compared to non-smokers $(0.48 \%)$. The study done by Pomp et $\mathrm{al}^{9}$ and Cheng et al ${ }^{10}$ also elicited a higher risk of DVT in smokers as compared to non-smokers due to elevated fibrinogen and thrombin levels in smokers.

\section{Correlation between BMI and DVT}

Average BMI of the study population was 21.06, whereas the average BMI of the patients who developed DVT was higher at 29.875 , which is similar to the study done by Heit JA et al, ${ }^{11}$ who found that VTE was associated with BMI $>30 \mathrm{~kg} / \mathrm{m}^{2}$. Increase in abdominal fat limit venous return and obesity causes thrombosis via leptin, increased activity of the coagulation cascade and decreased fibrinolysis. There may be increased inflammation, oxidative stress and endothelial dysfunction as postulated by Van Guilder et al. ${ }^{12}$

\section{Association of Malignancy with DVT}

The incidence of post-op DVT among patients with malignancy in this study was $4.54 \%$ as compared to $0.86 \%$ in patients without any malignancy. Falanga et $\mathrm{al}^{13}$ and Stein et al ${ }^{14}$ also showed cancer patients to be at a higher risk for DVT development. Venous compression secondary to tumour growth, cancer-associated thrombocytosis, immobility, indwelling central lines and chemotherapy or radiation therapy increase the possibility of VTE.

\section{Hyperlipidaemia and DVT}

Incidence of DVT in hyperlipidaemic patients was $6.67 \%$ as compared to a low $0.36 \%$ in normolipidaemic patients. This is in accordance with the study conducted by Vaya et al ${ }^{15}$ and Kawasaki et al.16 Elevated plasma levels of PAI-1, ThrombinActivatable Fibrinolysis Inhibitor (TAFI), vWF, coagulation factors VIII, VII and XIII and fibrinogen, TF, endothelial cell microparticles and decreased protein $C$ levels are observed in hyperlipidaemic patients.

\section{Duration of Surgery}

In this study, the average of the duration of surgery in the patient population was 1.99 hours, whereas it was 2.91 hours in those who developed DVT post-operatively. These findings are similar to those found in the study conducted by Kim et al, ${ }^{17}$ who concluded that an increase in duration of surgery was directly associated with an increase in the risk for VTE.

This can be explained by the fact that surgery is accompanied by a transient, low-level hypercoagulable state, mediated by the release of tissue factor, which is marked by a rise in thrombin activation markers shortly after the procedure begins. Increased levels of PAI-1 are also associated with a decrease in fibrinolytic activity on the first postoperative day. 


\section{Type of Anaesthesia and DVT}

In this study, all the eight patients which developed DVT postsurgery, all were operated under general anaesthesia. The patients who received spinal and epidural anaesthesia did not show DVT post-operatively.

Narani.18 showed higher risk of DVT in patients who received GA due to marked increase in tissue factor, $v W F$, plasminogen activator inhibitor-1 (PAI-1) and tissue plasminogen activator, resulting in a hypercoagulable and hypofibrinolytic state post-operatively in patients receiving GA.

\section{Postoperative Immobilisation and DVT}

The average duration of immobilisation post-surgery in this study was 1.85 days, whereas the average was higher in patients diagnosed with DVT being 3 days. Ahmed et al 19 stated prolonged immobilisation as a risk factor for developing DVT. Immobilisation leads to stasis of the blood column in the soleal veins and behind the valve cusps due to inactivity of the calf muscle pump.

\section{Type of Surgery Performed}

Three of the four patients who underwent a colonic surgery developed DVT. Out of all the patients who underwent colonic surgeries, $40 \%$ developed DVT. This is synergistic to the study done by Schaub et al ${ }^{20}$ who showed $58.3 \%$ incidence in DVT after colonic surgery.

Appendectomies, cholecystectomies and hernia repair surgeries, etc. were associated with no risk of DVT development. Samama et $\mathrm{al}^{21}$ showed minor abdominal surgeries, such as appendicectomy carry a relatively low VTE risk of $0.1 \%$. Riber et al $^{22}$ showed the incidence of VTE associated with day surgery for inguinal hernia repair is a modest $0.04 \%$.

Although MRM is associated with a low risk of developing DVT postoperatively, our patient might have developed DVT because of higher BMI, associated hyperlipidaemia and a positive history of OCP intake.

\section{Clinical Presentations in DVT}

Limb pain had a sensitivity of $100 \%$, but a low specificity of $64.21 \%$ in this study. Limb swelling had a low sensitivity and specificity of $50 \%$ and $61.25 \%$. The sensitivity and specificity rates of this study were similar to those obtained by Anand et al. ${ }^{23}$ According to Tovey et al, ${ }^{24}$ the clinical diagnosis of DVT is unreliable.

\section{Homans' Sign}

A clinical sign frequently used to suspect DVT in symptomatic patients yielded a low sensitivity of $50 \%$ and specificity of $51.875 \%$ in this current study. This was in accordance to the study done by Riddle and Wells. ${ }^{25}$ who suggested Homans' sign has essentially no diagnostic value with sensitivities around $50 \%$.

A positive Homans' sign can also be elicited in calf muscle spasm, neurogenic leg pain, ruptured Baker's cyst and cellulitis. Thus, this sign is generally unreliable as a clinical sign of DVT.

\section{Role of D-Dimer in Diagnosis of DVT}

The sensitivity and specificity of D-dimer in diagnosing DVT in this study was $75 \%$ and $56.09 \%$ respectively. This value corresponds to the sensitivity (84\%) and specificity (50\%) values obtained by Sartori et al. ${ }^{26}$ Thus, D-dimer has a low specificity in diagnosis of DVT. It has a better negative predictive value, but alone it does not exclude isolated distal DVT.

D-dimer (a product of fibrin proteolysis by plasmin) can also be elevated as a response to injury in patients undergoing surgical procedures or trauma, as part of the normal physiologic changes during pregnancy and as a part of the pathophysiologic process in patients with cancer or thrombotic disorders.

\section{Duplex USG in Diagnosis of DVT}

Duplex USG had a high sensitivity of $100 \%$ and high specificity of $99.21 \%$ in the diagnosis of DVT in the patients studied. Oliveira et al 27 in their study showed a sensitivity of $100 \%$ and specificity of $98 \%$ for DVT diagnosis.

Duplex ultrasonography remains the dominant diagnostic test of choice for the detection of DVT. It has almost completely replaced venography because of its accuracy, lack of radiation, portability, non-invasiveness and relative cost-effectiveness. In addition, ultrasound has the ability to distinguish among nonvascular pathologic processes, such as inguinal adenopathy, Baker's cyst, abscess and haematoma.

\section{CONCLUSION}

From this study, it can thus be concluded that the incidence of DVT is less in Asia as compared to the West. DVT mainly affects the older age groups and the risk progressively increases with advancing common after 50 years of age. The incidence of DVT is slightly higher in males and smokers. Females on OCPs are at greater risk.

Hyperlipidaemia, BMI $>25 \mathrm{~kg} / \mathrm{m}^{2}$ are significant risk factors for DVT development. Longer duration of surgery under GA and postoperative immobilisation $>48$ hours increase the chances of DVT after surgery. Thus, early ambulation is to be encouraged post-operatively.

DVT incidence is higher after colonic surgeries and surgeries for malignancy. Appendicectomy, hernia repairs, breast surgeries are at lowest risk.

Pain, swelling and calf tenderness are the common clinical features of DVT. High variability and lack of specificity limit the role of clinical examination in diagnosing a case of DVT. Ddimer has low sensitivity and specificity which limits its use in patients with suspected DVT. Duplex USG has a high sensitivity and specificity and thus remains the dominant diagnostic test of choice for the detection of DVT.

Patients aged $>65-70$ years, females on OCPs, BMI $>30$ $\mathrm{kg} / \mathrm{m}^{2}$, patients having an elevated lipid profile, smokers, previous or family history of VTE, patients with malignancy, duration of surgery $>2.9$ hours, (Mainly colonic surgeries) patients receiving GA, postoperative immobilisation $>3$ days should receive the use of IPC combined with a LDUH or LMWH for the thromboprophylaxis if no bleeding risk is present according to the latest edition of the ACCP guidelines for the management of VTE.

\section{REFERENCES}

1. Murthy SSR, Rao PLNG, Sodhi JS, et al. Post-operative deep vein thrombosis. J Asso Phy Ind 1979;27:239-45.

2. Stein PO, Patel KC, Kalra NK, et al. Deep venous thrombosis in a general hospital. Chest 2002;122(3):9602 . 
3. Smith BR, Diniz S, Stamos M, et al. Deep venous thrombosis after general surgical operations at a university hospital: two-year data from the ACS NSQIP. Arch Surg 2011;146(12):1424-7.

4. Aiach M, Gandrille S, Emmerich J. A review of mutations causing deficiencies of antithrombin, protein $\mathrm{C}$ and protein S. Thromb Haemost 1995;74(1):81-9.

5. Rakesh RA, Priyatham K, Ramprakash, et al. A change in trend of age related distribution of DVT. Ind J Appl Rsch 2015;5(9):2249-555.

6. Silverstein MD, Heit JA, Mohr DN, et al. Trends in the incidence of deep vein thrombosis and pulmonary embolism: a 25 year population-based study. Arch Intern Med 1998;158(6):585-93.

7. Legato MJ. Principles of gender-specific medicine. Academic Press 2009; 2nd ed:798.

8. Vlieg A Van H, Helmerhorst FM, Vandenbroucke JP, et al. The venous thrombotic risk of oral contraceptives, effects of oestrogen dose and progestogen type: results of the MEGA case-control study. BMJ 2009;339:b2921.

9. Pomp ER, Rosendaal FR, Doggen CJ. Smoking increases the risk of venous thrombosis and acts synergistically with oral contraceptive use. Am J Haematol 2008;83(2):97-102.

10. Cheng Y-J, Liu Z-H, Yao F-J, et al. Current and former smoking and risk for venous thromboembolism: a systematic review and meta-analysis. PLoS Med 2013;10(9):e1001515.

11. Heit JA, Petterson TM, Bailey KR, et al. Risk factors for venous thromboembolism among patients hospitalized for acute medical illness: a population-based case-control study. J Thromb Haemost 2005;3(8):1611.

12. Van Guilder GP, Hoetzer GL, Greiner JJ, et al. Metabolic syndrome and endothelial fibrino-lytic capacity in obese adults. Am J Physiol Regul Integr Comp Physiol 2008;294(1):R39-44.

13. Falanga A, Zacharski L. Deep vein thrombosis in cancer: the scale of the problem and approaches to management. Ann Oncol 2005;16(5):696-701.

14. Stein PD, Beemath A, Meyers FA, et al. Incidence of venous thromboembolism in patients hospitalized with cancer. Am J Med 2006;119(1):60-8.
15. Vaya A, Mira Y, Ferrando F, et al. Hyperlipidaemia and venous thromboembolism in patients lacking thrombophilic risk factors. $\mathrm{Br} \quad \mathrm{J}$ Haematol 2002;118(1):255-9.

16. Kawasaki T, Kambayashi J, Sakon M. Hyperlipidemia: a novel aetiologic factor in deep vein thrombosis. Thromb Res 1995;79(2):147-51.

17. Kim JYS, Khavanin N, Rambachan A, et al. Surgical duration and risk of venous thromboembolism. JAMA Surg 2015;150(2):110-7.

18. Narani KK. Deep vein thrombosis and pulmonary embolism-prevention, management, and anaesthetic considerations. Indian J Anaesth 2010;54(1):8-17.

19. Ahmed MM, Akbar DH, Al-Shaikh AR. Deep vein thrombosis at king abdul aziz university hospital. Saudi Med J 2000;21(8):762-4.

20. Schaub N, Duckert F, Fridrich $R$, et al. Incidence of postoperative deep vein thrombosis in general surgical and urological patients and investigation by means of the 125I-fibrinogen test in 95 patients withoug prophylaxis (author's transl). Langenbecks Arch Chir 1975;340(1):2334.

21. Samama CM, Albaladejo P, Benhamou D, et al. Venous thromboembolism prevention in surgery and obstetrics: clinical practice guidelines. Eur J Anaesthesiol 2006;23(2):95-116.

22. Riber C, Alstrup N, Nymann T, et al. Postoperative thromboembolism after day-case herniorrhaphy. $\mathrm{Br} \mathrm{J}$ Surg 1996;83(3):420-1.

23. Anand SS, Wells PS, Hunt D, et al. Does this patient have deep vein thrombosis? JAMA 1998;279(14):1094-9.

24. Tovey C, Wyatt S. Diagnosis, investigation, and management of deep vein thrombosis. BMJ 2003;326(7400):1180-4.

25. Riddle DL, Wells PS. Diagnosis of lower-extremity deep vein thrombosis in outpatients. Phys Ther 2004;84(8):729-35.

26. Sartori M, Cosmi B, Legnani C, et al. The wells rule and Ddimer for the diagnosis of isolated distal deep vein thrombosis. J Thromb Haemost 2012;10(11):2264-9.

27. Oliveira A De, França GJ, Vidal EA, et al. Duplex scan in patients with clinical suspicion of deep venous thrombosis. Cardiovascular Ultrasound 2008;6(1):53. 